\title{
Effects of Trabecular Meshwork Width and Schlemm's Canal Area on Intraocular Pressure Reduction in Glaucoma Patients
}

\author{
Hyun Woo Chung, Ji-Hye Park, Chungkwon Yoo, Yong Yeon Kim \\ Department of Ophthalmology, Korea University College of Medicine, Seoul, Korea
}

\begin{abstract}
Purpose: To evaluate the effects of baseline trabecular meshwork (TM) and Schlemm's canal (SC) microstructures on intraocular pressure (IOP) reduction amount in treatment-naïve patients with primary open-angle glaucoma (POAG).

Methods: A total 69 eyes of POAG patients who had not been treated with IOP-lowering agent were enrolled in this retrospective study. The patients had been prescribed topical IOP-lowering agent and used it for 1 year. The morphologic features of the TM and SC were collected using anterior segment module of spectral-domain optical coherence tomography with enhanced depth imaging at baseline. Images of the nasal and temporal corneoscleral limbus were obtained with serial horizontal enhanced depth imaging B-scans and TM width and SC area were measured in each scan. We investigated the effects of baseline TM and SC microstructures on IOP reduction amount.

Results: The baseline IOP of 69 glaucomatous eyes was $17.9 \pm 3.8 \mathrm{mmHg}$, and the mean amount of IOP reduction was $3.5 \pm 2.1$ $\mathrm{mmHg}$ after 1 year. Mean TM widths of nasal and temporal sector were $470.33 \pm 80.05$ and $479.74 \pm 79.59 \mu \mathrm{m}$, respectively. SC area was measured as 4,818.50 $\pm 1,464.28,4,604.23 \pm 1,567.73 \mu \mathrm{m}^{2}$ at nasal sector and temporal sector, respectively. The correlation analysis revealed a positive correlation between $\mathrm{SC}$ area and average amount of IOP reduction, indicating that the larger baseline SC area, the greater the IOP drop with topical IOP-lowering agents. However, no correlation was found between TM width and IOP lowering amount in patients with POAG.

Conclusions: The baseline SC area showed positive correlation with the IOP reduction amount in patients with POAG. This finding suggests that the SC area can be a clinical parameter to predict the IOP reduction amount before using IOP-lowering agents in POAG patient.
\end{abstract}

Key Words: Anterior segment optical coherence tomography, Aqueous outflow, Glaucoma, Schlemm's canal, Trabecular meshwork

Received: January 6, 2021 Final revision: May 11, 2021

Accepted: June 18, 2021

Corresponding Author: Ji-Hye Park, MD, PhD. Department of Ophthalmology, Korea University Ansan Hospital, 123 Jeokgeum-ro, Danwon-gu, Ansan 15355, Korea. Tel: 82-31-412-5160, Fax: 82-31-412-4267, E-mail: jennypark8321@gmail.com

This study was presented at the 124th Annual Meeting of the Korean Ophthalmological Society in Seoul, Korea on October 30 to November 1, 2020.
Glaucoma is defined as a progressive optic neuropathy characterized by structural loss and visual field defects. It is one of the leading causes of irreversible blindness [1]. Although the exact mechanism of glaucomatous damage is unknown, the most important risk factor for glaucoma development and progression is increased intraocular pressure (IOP) [2]. It is widely accepted that the formation and outflow of aqueous humor plays an important role in IOP 
regulation. Most of the aqueous humor, secreted by ciliary body, exits the anterior chamber through trabecular meshwork (TM) which leads to Schlemm's canal (SC). Eventually, through episcleral veins, aqueous humor joins the blood circulation to maintain the dynamic balance [3]. Elevated IOP is known to be affected by several factors, but the increase in outflow resistance due to changes in anatomical or histological structure is considered to be most important $[4,5]$.

Anterior segment imaging techniques, such as ultrasound biomicroscopy and anterior segment optical coherence tomography (AS-OCT), have been used to evaluate the structure of anterior chamber angle noninvasively $[6,7]$. Several studies have scanned the limbal area by AS-OCT and showed the usefulness of AS-OCT for visualizing the microstructure of aqueous humor outflow pathway. Kagemann et al. [8] showed that acute IOP elevation significantly reduces the SC cross-sectional area in healthy eyes by using spectral-domain optical coherence tomography (OCT). Hong et al. [9] investigated the features of SC in patients with open-angle glaucoma with spectral-domain OCT and reported a decreased SC area compared with normal eyes. Furthermore, Masis et al. [10] showed that TM width was significantly different between open and closed angle patients.

Recently, Qi et al. [11] reported that thinner TM and smaller SC vertical diameter are anatomical risk factors for early IOP elevation after cataract surgery in highly myopic eyes. They suggested that eyes with these anatomical features are less capable of handling aqueous drainage during early postoperative period and stressed the importance of evaluating these anatomical structures before cataract surgery. However, to the best of our knowledge, there are no reports evaluating the effects of baseline TM and $\mathrm{SC}$ microstructures on IOP reduction amount or IOP fluctuation after using topical IOP-lowering agent. Therefore, we investigated the baseline morphology of TM width and SC area of patients with primary open-angle glaucoma (POAG) and explored their possible relationship with IOP reduction amount.

\section{Materials and Methods}

We reviewed the database of POAG patients who visited the glaucoma clinic at Korea University Ansan Hospital from March 2017 to June 2019, and we retrieved the data of those who had not been treated with IOP-lowering agent when they were first diagnosed as POAG. This retrospective study was conducted with approval from the institutional review board of the Korea University Ansan Hospital (2020AS0029) andt the requirement for informed consent was waived due to the retrospective design of the study. The principles outlined in the Declaration of Helsinki were followed throughout the study.

Treatment-naïve patients with POAG who were first diagnosed at Korea University Ansan Hospital and had been prescribed topical IOP-lowering agents and used it for 1 year were eligible for inclusion in this study. All patients underwent ophthalmic examinations, including best-cor-
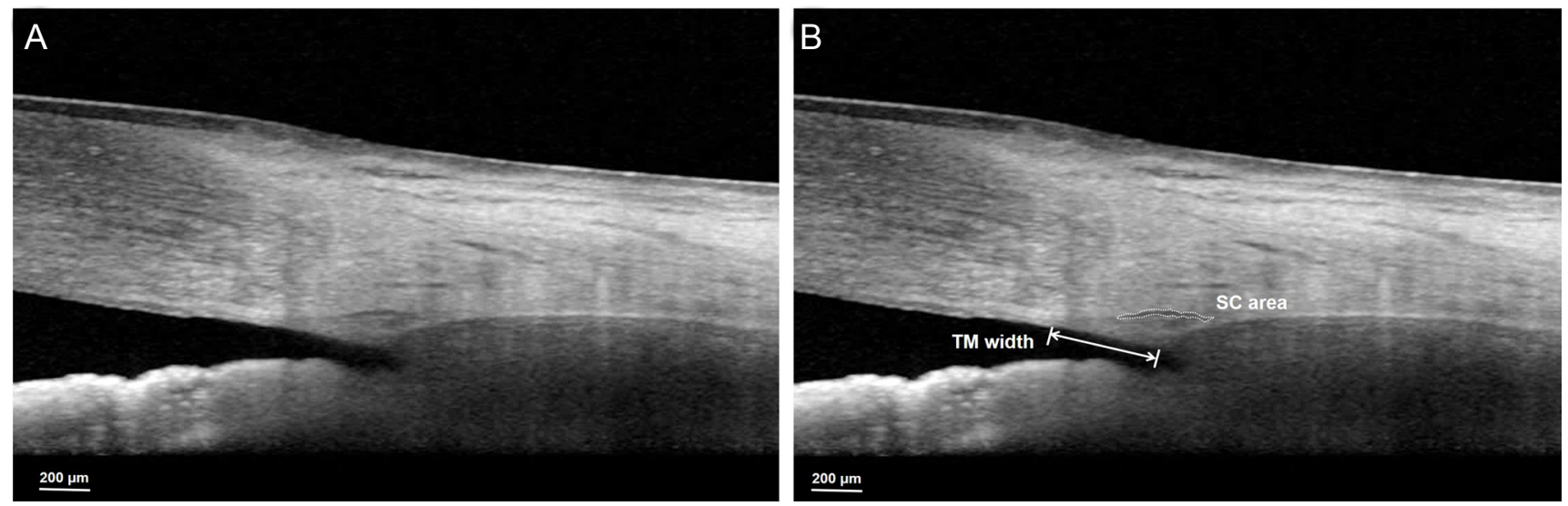

Fig. 1. The measurements of Schlemm's canal (SC) area and trabecular meshwork (TM) width. (A) Original image and (B) after identification of each parameter. TM width was manually measured as the distance from the scleral spur to the Schwalbe line. The SC area, identified as a horizontally oval- or elliptic-shaped black translucent space, was manually measured using ImageJ software. In this case, the TM width and SC area were $421 \mu \mathrm{m}$ and $4,797.31 \mu \mathrm{m}^{2}$, respectively. 
rected visual acuity, refraction, slit-lamp biomicroscopy, Goldmann applanation tonometry, axial length by IOLMaster (Carl Zeiss Meditec, Jena, Germany), central corneal thickness, visual field testing by Swedish interactive threshold algorithm and 24-2 test pattern of the Humphery Field Analyzer (Zeiss-Humphrey, San Leandro, CA, USA), and gonioscopy. POAG was defined when they showed an open anterior chamber angle on gonioscopy, reproducible Humphrey SITA 24-2 visual field test abnormality (Zeiss-Humphrey) compatible with glaucoma, and a glaucomatous appearance of the optic disc. The patients were examined 1, 3, 6, 9, and 12 months after starting to use topical IOP-lowering agents, and their IOPs were checked at every visit.

Patients were excluded if they met any of the following criteria: (1) history of using IOP-lowering agent, (2) invisible TM in any quadrant on static gonioscopy, (3) presence of retinal disease, (4) refractive error exceeding spherical equivalent of 6 diopters or astigmatism of 3 diopters, or (5) previous ocular surgery except uncomplicated cataract surgery more than 1 year before recruitment.

\section{Measurement of TM width and SC area}

A single experienced technician performed the anterior segment module of spectral-domain OCT (Heidelberg Spectralis OCT; Heidelberg Engineering, Heidelberg, Germany) with enhanced depth imaging (EDI) at baseline. Images of the nasal and temporal corneoscleral limbus were obtained with serial horizontal EDI B-scans. Patients with an incomplete set of EDI OCT B-scans or with poor quality scans in which the TM and SC could not be reliably recognized were excluded from the analysis. The TM width and SC area were measured in each scan by a single observer who was blinded to the patients' glaucoma status. The TM width was defined as the distance from the scleral spur to the Schwalbe line and was measured manually using the built-in caliper of the software provided in the OCT instrument. The location of the scleral spur was selected as the point where there was a change in the curvature of the inner surface of the angle wall, often presenting as an inward protrusion of the sclera. The SC, identified as a horizontally oval- or elliptic-shaped black translucent space, was measured by manually delineating the SC lumen using ImageJ software ver. 1.52 (National Institutes of
Table 1. Demographics and clinical characteristics for study subjects $(n=69$ eyes $)$

\begin{tabular}{lc}
\hline Characteristics & Value \\
\hline Age & $52.49 \pm 14.10$ \\
Sex (female : male) & $36: 33$ \\
Laterality (right : left) & $18: 51$ \\
Axial length (mm) & $24.44 \pm 1.37$ \\
Anterior chamber depth (mm) & $3.40 \pm 0.37$ \\
Spherical equivalent (diopter) & $-1.71 \pm 2.57$ \\
IOP (mmHg) & \\
Baseline & $17.9 \pm 3.8$ \\
After 1 mon & $14.6 \pm 2.8$ \\
After 3 mon & $14.2 \pm 3.0$ \\
After 6 mon & $14.6 \pm 3.1$ \\
After 9 mon & $14.4 \pm 2.9$ \\
After 1 yr & $14.3 \pm 3.0$ \\
Post-medication average IOP* & $14.4 \pm 2.7$ \\
Post-medication IOP SD & $1.4 \pm 0.8$ \\
Post-medication IOP range* & $2.9 \pm 1.7$ \\
Mean IOP reduction & $3.5 \pm 2.1$ \\
Percentage mean IOP & $18.76 \pm 9.03$ \\
reduction (\%) & $-4.44 \pm 4.04$ \\
Mean deviation (dB) & $91.22 \pm 10.36$ \\
Visual field index (\%) & \\
Average retinal nerve fiber & \\
layer thickness ( $\mu$ m) & \\
Medications & \\
Prostaglandin analogues & \\
FDCD (carbonic anhydrase & Alpha agonist \\
\hline
\end{tabular}

Values are presented as mean $\pm \mathrm{SD}$ or number

$\mathrm{IOP}=$ intraocular pressure; $\mathrm{SD}=$ standard deviation; $\mathrm{FDCD}=$ fixed-dose combination drug.

*Defined as the mean value of IOPs at 1, 3, 6, 9 months and 1 year; ${ }^{\dagger}$ Defined as the SD of the mean IOP within of IOPs at 1 , $3,6,9$ months and 1 year; ${ }^{\star}$ Calculated as the difference between maximum and minimum IOP values after using IOP-lowering medication; ${ }^{\S}$ Calculated by subtracting the post average IOP from the baseline IOP.

Health, Bethesda, MD, USA) (Fig. 1A, 1B).

\section{Statistical analysis}

Statistical analysis was performed using IBM SPSS Statistics ver. 21.0 (IBM Corp., Armonk, NY, USA). The nor- 
mality of distribution was verified using the Shapiro-Wilk normality test. Pearson correlation analysis was used to evaluate the association between baseline AS-OCT parameters and IOP parameters. In a subgroup analysis, the patients were divided into two groups according to their average IOP reduction amount, and the baseline ocular parameter and AS-OCT parameter were compared with independent $t$-test. A $p$-value $<0.05$ was considered statistically significant.

Table 2. Baseline anterior segment parameter

\begin{tabular}{lc}
\hline Parameter & Value \\
\hline Trabecular meshwork width $(\mu \mathrm{m})$ & \\
Nasal sector & $470.33 \pm 80.05$ \\
Temporal sector & $479.74 \pm 79.59$ \\
Average & $478.32 \pm 74.02$ \\
Schlemm's canal area $\left(\mu \mathrm{m}^{2}\right)$ & \\
Nasal sector & $4,818.50 \pm 1,464.28$ \\
Temporal sector & $4,604.23 \pm 1,567.73$ \\
Average & $4,754.14 \pm 1,354.08$ \\
\hline
\end{tabular}

Values are presented as mean \pm standard deviation.

\section{Results}

A total of 69 glaucomatous eyes were enrolled. The study included 33 males and 36 females (Table 1). Mean age of participants was $52.49 \pm 14.10$ years old. The baseline IOP of participants was $17.9 \pm 3.8 \mathrm{mmHg}$, and the mean amount of IOP reduction was $3.5 \pm 2.1 \mathrm{mmHg}$ after 1 year. Most of the patients were treated with prostaglandin analogues, following with carbonic anhydrase inhibitor/timolol fixed combination drug, prostaglandin/timolol fixed combination drug, and alpha agonist drug.

Table 2 shows the baseline TM width and SC area of included subjects. Mean TM width of nasal and temporal sector was $470.33 \pm 80.05$ and $479.74 \pm 79.59 \mu \mathrm{m}$, respectively. SC area was measured as $4,818.50 \pm 1,464.28$, $4,604.23 \pm 1,567.73 \mu \mathrm{m}^{2}$ at nasal sector and temporal sector. There was no significant difference between the nasal and temporal sector measurements for the TM width and SC area (TM width, $p=0.157$; SC area, $p=0.512$ ).

The association between baseline anterior segment parameter and IOP parameter is shown in Table 3. The correlation analysis revealed a positive correlation between $\mathrm{SC}$ area and average amount of IOP reduction, indicating that the larger baseline SC area, the greater the IOP drop with topical IOP-lowering agents. However, no correlation

Table 3. Pearson correlation coefficient between baseline anterior segment parameters and IOP parameters

\begin{tabular}{|c|c|c|c|c|c|}
\hline & & $\begin{array}{l}\text { Mean IOP } \\
\text { reduction }\end{array}$ & $\begin{array}{l}\text { Mean IOP reduction } \\
\text { percentage }\end{array}$ & $\begin{array}{l}\text { Post-medication } \\
\text { IOP SD }\end{array}$ & $\begin{array}{l}\text { Post-medication } \\
\text { IOP range }\end{array}$ \\
\hline \multicolumn{6}{|c|}{ Trabecular meshwork width } \\
\hline \multirow[t]{2}{*}{ Nasal sector } & $r$ & 0.153 & 0.151 & -0.005 & -0.019 \\
\hline & $p$-value & 0.217 & 0.223 & 0.968 & 0.876 \\
\hline \multirow[t]{2}{*}{ Temporal sector } & $r$ & 0.206 & 0.193 & 0.055 & 0.050 \\
\hline & $p$-value & 0.099 & 0.124 & 0.663 & 0.694 \\
\hline \multirow[t]{2}{*}{ Average } & $r$ & 0.180 & 0.181 & -0.007 & -0.010 \\
\hline & $p$-value & 0.139 & 0.136 & 0.953 & 0.937 \\
\hline \multicolumn{6}{|c|}{ Schlemm's canal area } \\
\hline \multirow[t]{2}{*}{ Nasal sector } & $r$ & 0.261 & 0.206 & 0.066 & 0.057 \\
\hline & $p$-value & 0.033 & 0.095 & 0.598 & 0.646 \\
\hline \multirow[t]{2}{*}{ Temporal sector } & $r$ & 0.241 & 0.237 & 0.136 & 0.107 \\
\hline & $p$-value & 0.053 & 0.057 & 0.279 & 0.395 \\
\hline \multirow[t]{2}{*}{ Average } & $r$ & 0.285 & 0.238 & 0.080 & 0.062 \\
\hline & $p$-value & 0.018 & 0.049 & 0.513 & 0.612 \\
\hline
\end{tabular}

$\mathrm{IOP}=$ intraocular pressure; $\mathrm{SD}=$ standard deviation. 
Table 4. Comparison of baseline anterior segment parameters and IOP parameters between subgroups divided by IOP reduction amount

\begin{tabular}{|c|c|c|c|}
\hline & $\begin{array}{l}\text { Small IOP } \\
\text { reduction group }(\mathrm{n}=19)\end{array}$ & $\begin{array}{c}\text { Large IOP } \\
\text { reduction group }(\mathrm{n}=50)\end{array}$ & $p$-value ${ }^{*}$ \\
\hline Baseline IOP (mmHg) & $15.3 \pm 1.9$ & $18.9 \pm 3.9$ & $<0.001$ \\
\hline Mean IOP reduction $(\mathrm{mmHg})$ & $1.2 \pm 0.6$ & $4.4 \pm 1.8$ & $<0.001$ \\
\hline Mean IOP reduction (\%) & $7.57 \pm 3.43$ & $23.01 \pm 6.48$ & $<0.001$ \\
\hline Axial length (mm) & $24.49 \pm 1.38$ & $24.32 \pm 1.38$ & 0.656 \\
\hline Anterior chamber depth (mm) & $3.39 \pm 0.36$ & $3.41 \pm 0.38$ & 0.842 \\
\hline Mean deviation $(\mathrm{dB})$ & $-3.77 \pm 4.05$ & $-4.70 \pm 4.04$ & 0.396 \\
\hline Visual field index (\%) & $93.05 \pm 10.34$ & $90.52 \pm 10.38$ & 0.368 \\
\hline $\begin{array}{l}\text { Average retinal nerve fiber layer } \\
\text { thickness }(\mu \mathrm{m})\end{array}$ & $82.00 \pm 16.25$ & $82.88 \pm 14.24$ & 0.826 \\
\hline Central corneal thickness $(\mu \mathrm{m})$ & $520.16 \pm 34.56$ & $524.22 \pm 43.15$ & 0.714 \\
\hline \multicolumn{4}{|l|}{ Trabecular meshwork width $(\mu \mathrm{m})$} \\
\hline Nasal sector & $453.47 \pm 91.39$ & $477.00 \pm 75.09$ & 0.282 \\
\hline Temporal sector & $458.33 \pm 91.15$ & $487.94 \pm 74.12$ & 0.182 \\
\hline Average & $460.05 \pm 92.85$ & $485.26 \pm 65.26$ & 0.288 \\
\hline \multicolumn{4}{|l|}{ Schlemm's canal area $\left(\mu \mathrm{m}^{2}\right)$} \\
\hline Nasal sector & $4,116.70 \pm 1,220.19$ & $5,096.29 \pm 1,470.63$ & 0.012 \\
\hline Temporal sector & $3,926.38 \pm 1,385.12$ & $4,863.83 \pm 1,569.02$ & 0.030 \\
\hline Average & $4,065.87 \pm 1,174.00$ & $5,015.68 \pm 1,335.93$ & 0.008 \\
\hline
\end{tabular}

Values are presented as mean \pm standard deviation.

$\mathrm{IOP}=$ intraocular pressure.

${ }^{*}$ Independent $t$-test.

was found between TM width and IOP lowering effect in patients with POAG. When the subjects were divided into two groups according to their average IOP reduction amount (small IOP reduction group, mean IOP reduction $<3 \mathrm{mmHg}$; large IOP reduction group, mean IOP reduction $\geq 3 \mathrm{mmHg}$ ), the large IOP reduction group showed higher baseline IOP (Table 4). Moreover, the baseline SC area was significantly larger in the large IOP reduction group compared to the small IOP reduction group.

\section{Discussion}

This study investigated the relationship between baseline TM width and SC area and the IOP reduction amount in treatment-naïve open-angle glaucoma patients. Our results indicate that the larger the baseline SC area, the greater the IOP drop with topical IOP-lowering agents. However, there was no correlation between baseline TM width and IOP reduction amount in open-angle glaucoma patients. These results imply that SC area may be more useful to predict the IOP reduction amount in POAG patients than TM width.

It is commonly known that there are two main pathways for aqueous humor outflow [3]. The trabecular outflow, also known as conventional pathway, accounts for up to $90 \%$ of the aqueous humor outflow, and the aqueous humor passes through the TM into Schlemm's canal and veins. The trabecular outflow resistance is regulated by the juxtacanalicular connective tissue and the inner wall of SC [12]. The other one, also known as unconventional pathway, is called uveoscleral outflow where the drainage of aqueous humor is mediated by ciliary muscle and other tissues $[13,14]$. Therefore, analyzing the anatomical structures involved in aqueous outflow could be useful in predicting the function of outflow facility and ultimately the IOP.

Previously, histologic studies have suggested the correlation between outflow facility and morphometric measurements of SC. Allingham and coworkers investigated the 
relationship between the pore density of SC inner wall endothelium and outflow facility in postmortem eyes, and reported a positive correlation as a result [15]. Further, Allingham et al. [16] reported in a subsequent study that the POAG eyes had significantly smaller SC cross sectional area and perimeter compared to the normal eyes. They suggested that reduction in SC dimensions may play an important role in decreasing outflow facility in POAG eyes. In addition, several studies evaluated the pressure-dependent changes of SC morphology in human eyes, and reported that increased IOP causes collapse of SC and this SC collapse is associated with an increase in outflow resistance [8,17-19]. Recently, anterior segment imaging technology has developed dramatically, and AS-OCT is now widely used to evaluate the anatomical structure of anterior chamber angle noninvasively. Several studies used AS-OCT for visualizing the microstructure of aqueous humor outflow pathway and revealed that SC area of eyes in glaucoma patients was smaller when compared to normal eyes [9,20,21]. Recent study by Qi et al. [11] reported that a smaller SC area and a thinner TM at baseline were associated with an early IOP elevation after cataract surgery in highly myopic eyes. They suggested that highly myopic eyes with small SC area may be less capable of dealing with aqueous drainage in the early postoperative period. This is consistent with our finding that large SC area at baseline was significantly associated with large amount of IOP reduction. Therefore, SC area may be a clinically useful parameter for evaluating physiologic changes of outflow pathway in glaucoma patient.

The TM is also an important site of outflow resistance in conventional outflow, and previous studies have investigated TM morphology in glaucoma patients as well $[10,22,23]$. Previous studies have suggested the relationship between structural alterations of TM and reduced aqueous outflow facility. In 1996, Stegman et al. [22] reported that TM width is shorter in juvenile POAG patients compared to normal subjects. Likewise, Yan et al. [23] investigated in vivo changes in SC and TM in eyes with POAG. They showed that TM thickness is thinner in POAG patients compared to normal subjects. Masis et al. [10] also reported that TM width is shorter in primary angle-closure glaucoma patients compared to POAG patients. However, unlike previous studies, there was no correlation between baseline TM width and IOP lowering effect in our study. Such inconsistent result between prior studies and ours may be attributed to differences in study design, study population, or measurement location. The possible assumptions why TM width was not a significant factor in our study can be considered as follows: (1) the microstructure of SC may better describe the aqueous outflow resistance or facility, (2) TM thickness, rather than TM width, may be a better parameter for reflecting aqueous humor outflow facility. Further study is needed to determine the role of baseline TM thickness in reducing IOP with topical medications in glaucoma patients.

There are several limitations in this study. First, relatively small number of eyes are included in this study. Second, the study population was limited to a single ethnic group. Previous studies have shown that anterior chamber angle parameters varies with ethnicity [24,25]. Therefore, it might be hard to generalize our results when dealing with other ethnic groups. Third, we only measured nasal and temporal corneoscleral limbus which may not represent the overall changes in TM and SC. Finally, we conducted this study using only two parameters which is SC area and TM width. There are possibilities that other parameter such as TM thickness might be more closely related to IOP reduction in POAG patients. Therefore, further studies are required to investigate other parameters which might affect IOP reduction in patients with POAG.

In conclusion, we found that $\mathrm{SC}$ area was larger in patients who had greater IOP reduction. In contrast, no correlation was found between TM width and IOP reduction amount. Therefore, assessment of SC microstructures by AS-OCT may be helpful to predict the amount of IOP reduction prior to the use of IOP-lowering medications in glaucoma patients.

\section{Conflict of Interest}

No potential conflict of interest relevant to this article was reported.

\section{References}

1. Nangia V, Jonas JB, George R, et al. Prevalence and causes of blindness and vision impairment: magnitude, temporal trends and projections in South and Central Asia. $\mathrm{Br} J$ Ophthalmol 2019;103:871-7. 
2. The Advanced Glaucoma Intervention Study (AGIS): 7. The relationship between control of intraocular pressure and visual field deterioration.The AGIS Investigators. $\mathrm{Am} \mathrm{J}$ Ophthalmol 2000;130:429-40.

3. Carreon T, van der Merwe E, Fellman RL, et al. Aqueous outflow: a continuum from trabecular meshwork to episcleral veins. Prog Retin Eye Res 2017;57:108-33.

4. Perlman JI, Delany CM, Sothern RB, et al. Relationships between $24 \mathrm{~h}$ observations in intraocular pressure vs blood pressure, heart rate, nitric oxide and age in the medical chronobiology aging project. Clin Ter 2007;158:31-47.

5. Sit AJ, Nau CB, McLaren JW, et al. Circadian variation of aqueous dynamics in young healthy adults. Invest Ophthalmol Vis Sci 2008;49:1473-9.

6. Dorairaj S, Liebmann JM, Ritch R. Quantitative evaluation of anterior segment parameters in the era of imaging. Trans Am Ophthalmol Soc 2007;105:99-110.

7. Leung CK, Li H, Weinreb RN, et al. Anterior chamber angle measurement with anterior segment optical coherence tomography: a comparison between slit lamp OCT and Visante OCT. Invest Ophthalmol Vis Sci 2008;49:3469-74.

8. Kagemann L, Wang B, Wollstein G, et al. IOP elevation reduces Schlemm's canal cross-sectional area. Invest Ophthalmol Vis Sci 2014;55:1805-9.

9. Hong J, Xu J, Wei A, et al. Spectral-domain optical coherence tomographic assessment of Schlemm's canal in Chinese subjects with primary open-angle glaucoma. Ophthalmology 2013;120:709-15.

10. Masis M, Chen R, Porco T, Lin SC. Trabecular meshwork height in primary open-angle glaucoma versus primary angle-closure glaucoma. Am J Ophthalmol 2017;183:42-7.

11. Qi J, He W, Lu Q, et al. Schlemm canal and trabecular meshwork features in highly myopic eyes with early intraocular pressure elevation after cataract surgery. Am J Ophthalmol 2020;216:193-200.

12. Civan MM, Macknight AD. The ins and outs of aqueous humour secretion. Exp Eye Res 2004;78:625-31.

13. Bill A. Uveoscleral drainage of aqueous humor: physiology and pharmacology. Prog Clin Biol Res 1989;312:417-27.
14. Bill A, Phillips CI. Uveoscleral drainage of aqueous humour in human eyes. Exp Eye Res 1971;12:275-81.

15. Allingham RR, de Kater AW, Ethier CR, et al. The relationship between pore density and outflow facility in human eyes. Invest Ophthalmol Vis Sci 1992;33:1661-9.

16. Allingham RR, de Kater AW, Ethier CR. Schlemm's canal and primary open angle glaucoma: correlation between Schlemm's canal dimensions and outflow facility. Exp Eye Res 1996;62:101-9.

17. Johnstone MA, Grant WG. Pressure-dependent changes in structures of the aqueous outflow system of human and monkey eyes. Am J Ophthalmol 1973;75:365-83.

18. Brubaker RF. The effect of intraocular pressure on conventional outflow resistance in the enucleated human eye. Invest Ophthalmol 1975;14:286-92.

19. Johnstone MA. Pressure-dependent changes in nuclei and the process origins of the endothelial cells lining Schlemm's canal. Invest Ophthalmol Vis Sci 1979;18:44-51.

20. Kagemann L, Wollstein G, Ishikawa H, et al. Identification and assessment of Schlemm's canal by spectral-domain optical coherence tomography. Invest Ophthalmol Vis Sci 2010;51:4054-9.

21. Imamoglu S, Sevim MS, Alpogan $\mathrm{O}$, et al. In vivo biometric evaluation of Schlemm's canal with spectral-domain optical coherence tomography in pseuduexfoliation glaucoma. Acta Ophthalmol 2016;94:e688-92.

22. Stegman Z, Sokol J, Liebmann JM, et al. Reduced trabecular meshwork height in juvenile primary open-angle glaucoma. Arch Ophthalmol 1996;114:660-3.

23. Yan X, Li M, Chen Z, et al. Schlemm's canal and trabecular meshwork in eyes with primary open angle glaucoma: a comparative study using high-frequency ultrasound biomicroscopy. PLoS One 2016;11:e0145824.

24. Lee RY, Chon BH, Lin SC, et al. Association of ocular conditions with narrow angles in different ethnicities. Am J Ophthalmol 2015;160:506-15.

25. Wang D, Qi M, He M, et al. Ethnic difference of the anterior chamber area and volume and its association with angle width. Invest Ophthalmol Vis Sci 2012;53:3139-44. 